\title{
High star formation rates in turbulent atomic-dominated gas in the interacting galaxies IC 2163 and NGC 2207
}

\author{
Bruce G. Elmegreen ${ }^{1}$, Michele Kaufman², Frédéric Bournaud ${ }^{3}$, Debra Meloy Elmegreen ${ }^{4}$, \\ Curtis Struck $^{5}$, Elias Brinks ${ }^{6}$, Stephanie Juneau ${ }^{3}$
}

\begin{abstract}
CO observations of the interacting galaxies IC 2163 and NGC 2207 are combined with $\mathrm{HI}, \mathrm{H} \alpha$ and $24 \mu \mathrm{m}$ to study the star formation rate (SFR) surface density as a function of the gas surface density. More than half of the high SFR regions are HI dominated. When compared to other galaxies, these HI-dominated regions have excess SFRs relative to their molecular gas surface densities but normal SFRs relative to their total gas surface densities. The HI-dominated regions are mostly located in the outer part of NGC 2207, where the HI velocity dispersion is high, $40-50 \mathrm{~km} \mathrm{~s}^{-1}$. We suggest that the star-forming clouds in these regions have envelopes at lower densities than normal, making them predominantly atomic, and cores at higher densities than normal because of the high turbulent Mach numbers. This is consistent with theoretical predictions of a flattening in the density probability distribution function for compressive, high Mach number turbulence.
\end{abstract}

Subject headings: ISM: molecules — Galaxies: star formation — Galaxies: interactions

\footnotetext{
${ }^{1}$ IBM Research Division, T.J. Watson Research Center, P.O. Box 218, Yorktown Heights, NY 10598; bge@watson.ibm.com

${ }^{2} 110$ Westchester Rd, Newton, MA 02458, USA; kaufmanrallis@icloud.com

${ }^{3}$ Laboratoire AIM-Paris-Saclay, CEA/DSM-CNRS-Université Paris Diderot, Irfu/Service d'Astrophysique, CEA Saclay, Orme des Merisiers, F-91191 Gif sur Yvette, France; frederic.bournaud@gmail.com; stephanie.juneau@cea.fr

${ }^{4}$ Department of Physics \& Astronomy, Vassar College, Poughkeepsie, NY 12604; elmegreen@vassar.edu

${ }^{5}$ Department of Physics \& Astronomy, Iowa State University, Ames, IA 50011; struck@iastate.edu

${ }^{6}$ University of Hertfordshire, Centre for Astrophysics Research, College Lane, Hatfield AL10 9AB, United Kingdom; e.brinks@herts.ac.uk
} 


\section{Introduction}

The galaxies IC 2163 and NGC 2207 are undergoing a grazing collision with perigalacticon $\sim 240$ Myr ago (Struck et al. 2005). The tidal force exerted on IC 2163 by NGC 2207 is prograde and nearly in-plane. It produces an intrinsically ocular structure (a cuspy oval) at mid-radius in IC 2163 and two tidal arms. A narrow ridge along the ocular appears to be a galactic-scale shock front with intense star formation, several super-star clusters including one measuring $2 \times 10^{5} M_{\odot}$ (Elmegreen et al. 2001), and an IR-to-radio continuum ratio $(S[8$ $\mu \mathrm{m}] / S[6 \mathrm{~cm}])$ that is a factor of 2 higher than elsewhere in the galaxies (Kaufman et al. 2012). HI observations (Elmegreen et al. 1995a) reveal photometric and kinematic major axes in IC 2163 that are nearly orthogonal, indicating high speed streaming motions. The tidal force exerted on NGC 2207 by IC 2163 is mostly perpendicular to NGC 2207, causing a velocity perturbation in NGC 2207 that is $\sim 200 \mathrm{~km} \mathrm{~s}^{-1}$ in places, and suggesting a warp with $15 \mathrm{kpc}$ vertical distortion (Elmegreen et al. 1995b).

Both galaxies have large HI velocity dispersions of $\sim 30-50 \mathrm{~km} \mathrm{~s}^{-1}$, and several large HI cloud complexes with masses of $10^{8} M_{\odot}$ or more (Elmegreen et al. 1993, 1995a). IC 2163 has an HI tidal bridge behind the eastern half of NGC 2207, and NGC 2207 has an HI spiral arm (visible also optically) in front of IC 2163 (see Figures in Elmegreen et al. 2000, where the HI contours are overlaid on an HST image).

NGC 2207 contains a peculiar region, Feature $i$, called a mini-starburst by Kaufman et al. (2012). This is the brightest source for either galaxy in radio continuum, $8 \mu \mathrm{m}, 24 \mu \mathrm{m}$ and $H \alpha$; it accounts for one-quarter of the total $24 \mu \mathrm{m}$ flux for both galaxies combined. At $\lambda 6$ $\mathrm{cm}$, the radio continuum luminosity of Feature $i$ is $13 \%$ of the radio continuum luminosity of the central starburst in M82. Soft X-ray emission was found by Mineo et al. (2014).

We observed NGC 2207/IC 2163 in CO(1-0) with the Atacama Large Millimeter Array (ALMA). The present paper is devoted to determining the spatially-resolved KennicuttSchmidt (Kennicutt \& Evans 2012) relation in this galaxy pair. We are interested in whether this relation on a scale of $2.4 \mathrm{kpc}$ diameter differs from that in other galaxies, and whether it is the same in both galaxies here. We combine the ALMA CO observations with our previous $\mathrm{HI}, 24 \mu \mathrm{m}$, and $H \alpha$ data to determine the star formation rate (SFR) surface density, $\Sigma_{\mathrm{SFR}}$, as a function of atomic, molecular, and total gas surface densities. Other properties of these CO observations will be discussed in Kaufman et al. (2016).

In what follows, Section 2 describes the observations, Section 3 gives the luminosities, SFRs, and surface densities of the selected regions, Section 4 shows the relations between $\Sigma_{\mathrm{SFR}}$ and the gas surface density, and Section 5 discusses the implications. 


\section{Observations}

IC 2163 and NGC 2207 were observed in 34 pointings using ALMA at the ${ }^{12} \mathrm{CO}(1-0)$ frequency of $115.27 \mathrm{GHz}(\lambda 2.6 \mathrm{~mm})$. We made naturally weighted maps of CO emission with a point spread function of $2.00^{\prime \prime} \times 1.52^{\prime \prime}(\mathrm{HPBW})$, beam position angle $68.5^{\circ}$, and channel width $10 \mathrm{~km} \mathrm{~s}^{-1}$. The rms noise per channel is $3.7 \mathrm{mJy}$ beam $^{-1}$ and a CO surface brightness of $1 \mathrm{Jy}^{\text {beam }^{-1}}$ corresponds to $T_{b}=30.7 \mathrm{~K}$. To select areas of genuine $\mathrm{CO}$ emission, we made a blanking mask by convolving this cube to $6^{\prime \prime} \times 6^{\prime \prime}$ HPBW, where the noise was 7.9 $\mathrm{mJy} /$ beam. Regions were left unblanked if they exceeded 2.5 times this rms noise for at least 2 adjacent channels in an area of at least 1 arc sec. This means that regions with line-of-sight $N\left(H_{2}\right)<2.45 M_{\odot} \mathrm{pc}^{-2}$ were blanked out. After correcting for primary beam attenuation, we made the $551 \times 301$ pixel $\times 64$ channel subcube used in this paper that contains all of the unblanked $\mathrm{CO}$ emission. In the subcube, the product of the rms noise and the channel width is equivalent to a line of sight $N\left(H_{2}\right)=3.27 \mathrm{M}_{\odot} \mathrm{pc}^{-2}$, which is a measure of our uncertainty. (The apertures A10, A16, A33, A38, and A39 discussed below have mean values of line-of-sight $N\left(H_{2}\right)$ below this because of blanked pixels.) The total integrated CO line flux in the subcube is $S(C O)=505 \mathrm{Jy} \mathrm{km} \mathrm{s}^{-1}$. For more details on the observing procedure, see Kaufman et al. (2016).

To convert $\mathrm{CO}$ emission into molecular mass $M\left(H_{2}\right)$, we use $X_{\mathrm{CO}}=1.8 \pm 0.3 \times 10^{20}$ $\mathrm{H}_{2} \mathrm{~cm}^{-2}\left(\mathrm{~K} \mathrm{~km} \mathrm{~s}^{-1}\right)^{-1}$ from Dame et al. (2001) and a distance $D=35 \pm 2.5 \mathrm{Mpc}$ from the NASA/IPAC Extragalactic Database (NED) for a Hubble constant of $H=73 \mathrm{~km} \mathrm{~s}^{-1} \mathrm{Mpc}^{-1}$ corrected for infall towards Virgo. The scale is 170 pc per arcsec. To convert surface density to face-on, we took inclinations of $i=40^{\circ}$ for IC 2163 and $35^{\circ}$ for NGC 2207 (Elmegreen et al. $1995 \mathrm{~b})$. We discuss the implications of different $X_{\mathrm{CO}}$ and $i$ in Section 4 .

The $24 \mu \mathrm{m}$ flux density, $S_{\nu}(24 \mu \mathrm{m})$, of Feature $i$ and the galaxies as a whole were measured from the MIPS 1 pBCD image obtained from the Spitzer archive in 2013; the resolution is $6^{\prime \prime}$. Other star-forming regions were measured on the HiRes deconvolution image $\left(\sim 1.9^{\prime \prime}\right.$ resolution) by Velusamy et al. (2008), who provided their HiRes $24 \mu \mathrm{m}$ data to us as a FITS image 1. According to Velusamy et al., the HiRes deconvolution conserves fluxes of point sources to better than $5 \%$. This uncertainty is in addition to the $5 \%$ uncertainty in the

\footnotetext{
${ }^{1}$ MIPS 1 BCD images from Spitzer prior to April 2007 suffer from two software bugs which cause the brightness of sources as bright as Feature $i$ to be underestimated. We compared our Spitzer $24 \mu \mathrm{m}$ MIPS 1 pBCD (level 2) image retrieved from the Spitzer archive in 2013 with the MIPS 1 image in Elmegreen et al. (2006). We found that Feature $i$ was the only source in NGC 2207/IC 2163 affected by the software bug, and that its flux density is a factor of 1.9 times greater than in Elmegreen et al. (2006) and Kaufman et al. (2012). Thus Feature $i$ accounts for $23 \%$, rather than $12 \%$, of the $24 \mu \mathrm{m}$ flux density from the galaxy pair. We use the corrected flux here.
} 
MIPS 1 photometric calibration. The HiRes deconvolution has about the same resolution as our CO data. This high resolution minimizes line-of-sight corrections for diffuse emission at $24 \mu \mathrm{m}$ that is not coincident with the star-forming region (Leroy et al. 2012). The absolute calibration of the $24 \mu \mathrm{m}$ term in the SFR leads to an uncertainty of about $25 \%$ in the absolute determination of the SFR (Leroy et al. 2012).

The HI is from VLA observations obtained in 1990 using a hybrid CnB array with an extended north arm to compensate for the low latitude of the source. The duration on source was 6 hours (for more details, see Elmegreen et al. 1995a). The map representing column density, $N(H I)$, comes from an HI cube with a channel width of $21 \mathrm{~km} \mathrm{~s}^{-1}$, rms noise 0.73 mJy beam ${ }^{-1}$, and FWHM of the point spread function equal to $13.5^{\prime \prime} \times 12^{\prime \prime}$. The product of the rms noise times the channel width is equivalent to a line-of-sight $N(H I)=0.84 M_{\odot}$ $\mathrm{pc}^{-2}$. Before creating the HI column density map, the HI data cube was masked in a similar manner to the CO data described above; none of our apertures contain blanks in HI.

The $\mathrm{H} \alpha$ data stem from narrowband $\mathrm{H} \alpha$ images and broadband $R$ images obtained by Deidre Hunter with the Lowell 1.1m telescope in 1999 (Elmegreen et al. 2001). Four H $\alpha$ images were combined for a total time of $3600 \mathrm{~s}$, and three $\mathrm{R}$ band images were combined for a total of $900 \mathrm{~s}$. The narrow filter excludes the redshifted [NII] line at $6583 \AA$, but includes the $6548 \AA$ line, which may contribute as much as $15 \%$ of the light for solar metallicity. Considering equation 1 below, a systematic $15 \%$ decrease in the $\mathrm{H} \alpha$ flux after removal of [NII] amounts to an average decrease in $\Sigma_{\mathrm{SFR}}$ of $5 \%$.

Figure 1 displays the chosen regions as circular apertures overlaid on the CO integrated intensity image, the HiRes $24 \mu \mathrm{m}$ image, and the $H \alpha$ image, which has a point spread function of $4.2^{\prime \prime} \times 3.6^{\prime \prime}$. The apertures were selected to have minimal overlap and to enclose regions with prominent $24 \mu \mathrm{m}, H \alpha, \mathrm{CO}$ and/or $\mathrm{HI}$ emission. They include three of the massive HI clouds in IC 2163 (I2, I3, I5) and four in NGC 2207 (N2, N3, N4, N6), even though some have little star formation. Figure 2 shows the HI contours on CO for IC 2163 (top) and NGC 2207 (bottom), where the separate HI contributions from each galaxy were determined from the line kinematics. For IC 2163 the lowest contour level is $5 \mathrm{M}_{\odot} \mathrm{pc}^{-2}$, which is equivalent to three times the rms noise over 2 channel widths. For NGC 2207 we omitted this contour level for clarity.

Table 1 compares global values of $M\left(H_{2}\right), M(H I)$, the $24 \mu \mathrm{m}$ flux density, $S_{\nu}(24 \mu m)$, and the $H \alpha$ flux, $S(H \alpha)$, for the fields displayed in Figure 1. For the values of $M\left(H_{2}\right)$, $S_{\nu}(24 \mu \mathrm{m})$, and $S(H \alpha)$, we chose a right ascension of $06^{\mathrm{h}} 16^{\mathrm{m}} 25.60^{\mathrm{s}}$ as the dividing line between IC 2163 and NGC 2207. For $M(H I)$, we included the tidal bridge of IC 2163 behind NGC 2207 and the spiral arm of NGC 2207 in front of IC 2163. The values listed under $M(H I)$ (total) include the HI emission outside the field in Figure 1; the values listed 
under $M(H I)$ (CO field) are only for the field in that figure. For IC 2163, the value of $S_{\nu}(24 \mu \mathrm{m})$ measured on the HiRes image is $84 \%$ of that measured on the MIPS 1 pBCD image (the HiRes image is less sensitive to faint extended emission).

Table 1 indicates that IC 2163 has a total molecular mass $M\left(H_{2}\right)=2.1 \times 10^{9} M_{\odot}$, which corresponds to an integrated CO line flux of $240 \mathrm{Jy} \mathrm{km} \mathrm{s}{ }^{-1}$, and NGC 2207 has $M\left(H_{2}\right)=2.3 \times 10^{9} M_{\odot}$ for a CO line flux of $265 \mathrm{Jy} \mathrm{km} \mathrm{s}^{-1}$. If we restrict the HI to the field of Figure 1, then the global ratio of molecular to atomic gas is 4 times greater in IC 2163 than in NGC 2207. For most of the 15 apertures in IC 2163 (except those on the massive HI clouds), the column density $N\left(H_{2}\right)$ dominates $N(H I)$, while for most of the 29 apertures in NGC 2207, $N(H I)>N\left(H_{2}\right)$. Thus, the eyelid shock in IC 2163 is currently more effective than the spiral arms of NGC 2207 in converting $\mathrm{HI}$ to $\mathrm{H}_{2}$. Also, the global ratio of $M\left(H_{2}\right)$ to $24 \mu \mathrm{m}$ flux density is $\sim 2$ times greater in IC 2163 than in NGC 2207, and the global ratio of $M\left(H_{2}\right)$ to $H \alpha$ flux is $\sim 3$ times greater in IC 2163 than in NGC 2207. These molecular excesses compared to star formation in IC 2163 are not as large as the molecular excesses compared to atomic gas as traced by HI. This difference suggests that the encounter has converted a high fraction of $\mathrm{HI}$ into $\mathrm{H}_{2}$ in IC 2163 but has not (yet) converted the additional molecules into stars.

\section{Star formation rates}

A combination of the continuum-subtracted $H \alpha$ image (Elmegreen et al. 2001) and the Spitzer MIPS $24 \mu \mathrm{m}$ image was used to obtain the SFR, following Kennicutt et al. (2009),

$$
\operatorname{SFR}\left(M_{\odot} \mathrm{yr}^{-1}\right)=5.5 \times 10^{-42}(L[H \alpha]+0.031 L[24 \mu m])
$$

where $L(H \alpha)$ and $L(24 \mu \mathrm{m})$ are in $\mathrm{erg} \mathrm{s}^{-1}$, and $L(24 \mu \mathrm{m})=\nu L_{\nu}$. We chose source apertures with a diameter of $14^{\prime \prime}=2.4 \mathrm{kpc}$. This choice was governed by the HI resolution and by the need to have a sufficiently large aperture to avoid stochastic effects from including too few $\mathrm{O}$ stars when using $\mathrm{H} \alpha$ as a star-formation tracer.

For the $24 \mu \mathrm{m}$ measurement of Feature $i$, we did a local background subtraction and applied an aperture flux correction factor of 1.61 from the MIPS Instrument Handbook2. It was not necessary to remove background for the CO or HI sources, nor for the other HiRes $24 \mu \mathrm{m}$ sources. For $H \alpha$, we did a global background subtraction but not a local subtraction because there would be contamination from adjacent sources in many cases.

\footnotetext{
${ }^{2}$ http://irsa.ipac.caltech.edu/data/SPITZER/docs/mips/mipsinstrumenthandbook/
} 
Foreground Galactic extinction $A_{\mathrm{V}}=0.238 \mathrm{mag}$ from NED (using Schlafly \& Finkbeiner 2011) gives an extinction at $H \alpha$ equal to $0.238 \mathrm{mag} / 1.28=0.186 \mathrm{mag}$. In addition, IC 2163 is affected by extinction from the foreground spiral arm of NGC 2207. Since the outer part of NGC 2207 is likely to be metal poor, we take $A_{\mathrm{V}}(\mathrm{mag})=(0.35 \pm 0.18) \times 10^{-21} \mathrm{~N}(\mathrm{HI})$ for this region (Elmegreen et al. 2001). This additional extinction is applied to 8 of the 15 regions in IC 2163. These 8 have the highest foreground $N(H I)$ and the largest corrections $\left(A_{\mathrm{V}}(H \alpha)>0.45 \mathrm{mag}\right)$, with an average $A_{\mathrm{V}}(H \alpha)=0.85 \pm 0.4 \mathrm{mag}$ from the foreground $\mathrm{HI}$ in NGC 2207. We assume that $H \alpha$ and $24 \mu \mathrm{m}$ emission from the foreground part of NGC 2207 are negligible.

Table 2 compiles the locations, luminosities, SFRs, $\Sigma_{\mathrm{SFR}}$, and surface densities for $\mathrm{H}_{2}$ and HI in all of the chosen apertures. The regions associated with massive HI clouds are indicated by the footnote $h$. The SFR is lower than 0.001 in the massive HI cloud apertures A10 and A33. This is below the limit suggested by Leroy et al. (2012) where the SFR can be reliably determined using the above method; the actual values do not contribute to the conclusions of this paper.

\section{Star formation - surface density correlation}

The conventional way to consider star formation on galactic scales is in terms of the correlation between $\Sigma_{\text {SFR }}$ and gas surface density (the "Kennicutt-Schmidt" relation), either for HI $\left(\Sigma_{\mathrm{HI}}\right), \mathrm{H}_{2}\left(\Sigma_{\mathrm{H} 2}\right)$, or the sum of these (Kennicutt \& Evans 2012).

Figure 3 shows these correlations. Figure 3(a) has the total gas relation, Figure 3(b) has $\mathrm{H}_{2}$ alone, 3(c) has $\mathrm{HI}$ alone, and 3 (d) shows $\Sigma_{\mathrm{SFR}}$ versus $\Sigma_{\mathrm{H} 2} / \Sigma_{\mathrm{HI}}$, the molecular ratio. Figure 3(d) is divided into three parts: where $\Sigma_{\mathrm{SFR}}>0.01$, the green points are for a molecular ratio less then 1 and the red points are for a molecular ratio greater than 1 . Where $\Sigma_{\mathrm{SFR}}<0.01$, the blue points are plotted for all molecular ratios. These colors have the same meaning in the other panels.

First consider the molecular relation in Figure 3(b). In the THINGS survey (Bigiel et al. 2008; Leroy et al. 2008), this was linear with a constant consumption time per CO molecule of 1-2 Gyr. This is the case here too, but only for the red and blue points, i.e., for the molecular-dominated gas at high SFR and all of the gas at low SFR. The HI-dominated gas at high SFR (green points) lies high off the linear molecular relation. The scatter in the molecular Kennicutt-Schmidt relation in Bigiel et al. (2008) is \pm 0.2 dex for $\Sigma_{\mathrm{SFR}}$, which is three times smaller than the displacement of the green points from the red+blue point correlation in Figure 3(b). The $\mathrm{H}_{2}$ consumption time for the HI-dominated gas is $\sim 300$ 
Myr, which is relatively fast compared to the time for the $\mathrm{H}_{2}$-dominated gas. The red circles around the points are for locations in the ocular rim of IC 2163.

The total-gas relation in Figure 3(a) has a consumption time for total gas of about 1 Gyr in regions with high SFRs (green and red points). This time is normal for molecular gas, but here it includes HI and also applies in HI-dominated regions (green points). The blue points (low SFR) have longer total-gas consumption times, between $\sim 3$ and 10 Gyr.

In Figure 3(c), the molecular-dominated regions (red points) have relatively low HI and the HI-dominated regions (green points) have relatively high HI. The values for the HI surface density in the HI-dominated, high SFR regions are unusually high: $\Sigma_{\mathrm{HI}}$ is greater than $20 M_{\odot} \mathrm{pc}^{-2}$ in many cases, which is twice as large as the saturated value of $\mathrm{HI}$ in the THINGS survey (Bigiel et al. 2008; Leroy et al. 2008).

What is peculiar about these relationships is the HI. If we ignore the HI-rich, high SFR regions (green points), then the molecular relation in Figure 3(b) is normal; the nearly constant $\Sigma_{\mathrm{HI}}$ up to $\sim 10 M_{\odot} \mathrm{pc}^{-2}$ for the high-SFR regions (red points) in Figure 3(c) is normal, and the steady increase in $\Sigma_{\text {SFR }}$ with molecular fraction in Figure 3(d) is normal. In these galaxies, however, there is a component of high HI column density gas where the SFR is high also. The HI is replacing some fraction of the molecules, giving a linear relation when the SFR is plotted against the total gas (Fig. 3(a)). Similarly, the HI-dominated regions lie off the normal linear law when the SFR is plotted versus only the molecular part of the gas (Fig. 3(b)). We also note that the molecular ratio $\Sigma_{H 2} / \Sigma_{H I}$ increases with decreasing $L(H \alpha) /[0.031 L(24 \mu m)]$ (not shown) suggesting more optical extinction in the star-forming cores when the molecular content is high.

Figure 4 shows the locations of these three types of regions on the sky using the same color scheme as in Figure 3. The HI-rich regions of high $\Sigma_{\mathrm{SFR}}$ are on the periphery of the galaxy that underwent the retrograde, perpendicular encounter (NGC 2207) and are globally coincident with the areas of high HI velocity dispersion in that galaxy (see Elmegreen et al. 1995a). The $\mathrm{H}_{2}$-rich regions of high $\Sigma_{\mathrm{SFR}}$ are mainly in the ocular ridge of IC 2163 where in-plane tidal forces produced a compression. The low $\Sigma_{\mathrm{SFR}}$ regions are scattered across both galaxies.

Feature $i$ in each panel (denoted by "i") has $\Sigma_{\mathrm{SFR}}$ at least a factor of 5 higher than in any other region. The molecular mass of Feature $i$ is $8 \times 10^{7} M_{\odot}$, which is not unusual for these $2.4 \mathrm{kpc}$ diameter apertures. In IC 2163, 11 of the 15 apertures have molecular masses greater than this (Table 2), and the mass in region A9 is 2.5 times greater. However, because of the high SFR, the molecular gas consumption time for Feature $i, \Sigma_{\mathrm{H} 2} / \Sigma_{\mathrm{SFR}}=50 \mathrm{Myr}$, is much shorter than for the other regions, and the total gas consumption time, $\left(\Sigma_{\mathrm{HI}}+\Sigma_{\mathrm{H} 2}\right) / \Sigma_{\mathrm{SFR}}=$ 
$118 \mathrm{Myr}$, is shorter too, as indicated by the positions of Feature $i$ relative to the dashed red lines in Figures 3(b) and (a), respectively.

A lower $X_{\mathrm{CO}}$ as suggested for some interacting galaxies and in Bournaud et al. (2015) would decrease the $\mathrm{H}_{2}$ mass and strengthen the conclusion that there are HI-rich star-forming regions with peculiar cloud structure. A higher $X_{\mathrm{CO}}$ would bring the $\mathrm{H}_{2}$ content of these regions in line with the CO-bright regions, but then $X_{\mathrm{CO}}$ would have to vary from cloud to cloud and be high primarily in the outer parts of NGC 2207, where the peculiar HI gas is. These outer parts are not so remote that low metallicities and an associated high $X_{\mathrm{CO}}$ are expected. Higher $X_{\mathrm{CO}}$ is also unlikely because the high velocity dispersion should lower the $\mathrm{CO}$ opacity by broadening the line, producing more $\mathrm{CO}$ emission per unit $\mathrm{H}_{2}$ molecule (Bournaud et al. 2015). Inclination effects would not seem to be causing the peculiar HI either. The suspected warp in NGC 2207 occurs about where this HI is, but correcting for that by lengthening the line-of-sight for gas emission only strengthens the conclusions because it lowers the average density for the observed column of HI and makes star formation slower, when in fact it is faster than normal. Changing the inclination slides points parallel to the lines of constant gas consumption time in the Kennicutt-Schmidt relation because it affects the deprojected areas used for the ordinate and abscissa equally.

A second version of Figure 4 was made (not shown) with all of the images convolved to $14^{\prime \prime} \times 14^{\prime \prime}$ resolution before the measurements were made inside the same apertures. This change had the effect of decreasing the fluxes used for the SFRs and $\Sigma_{\mathrm{H} 2}$ by factors between 0.5 and 0.9 , and that moved the points in the figure slightly down and to the left in a direction nearly parallel to the lines of constant consumption time. Considering the wings of the Gaussian, this is the expected reduction factor for a small source located somewhere in the aperture when the aperture diameter is the same as the FWHM of the point spread function in the convolved image. None of the conclusions of this paper were affected by this change.

\section{Discussion}

HI-dominated regions with high SFRs are unusual and could be related to the high turbulent speeds in this interacting pair. The mean HI velocity dispersion for the 16 green circles in N2207 (Fig. 4) is $42 \mathrm{~km} \mathrm{~s}^{-1}$, compared to $\sim 10 \mathrm{~km} \mathrm{~s}^{-1}$ in normal galaxies (Tamburro et al. 2009). An interaction perturbs the smooth circular flow of gas and causes deflected streams to intersect each other at high speeds, producing shocks and a turbulent cascade. Numerical simulations show this effect (Wetzstein et al. 2007; Bournaud et al. 2011a; Powell et al. 2013). Some turbulent energy in NGC 2207 may also come from excited vertical motions. 
High velocity dispersions in interacting systems were also reported by Kaufman et al. (1999), Rich et al. (2015) and others. In comparison, the THINGS galaxies and most others used for the conventional SFR-gas correlations are not interacting.

Stronger shocks imply a greater compressive component to the turbulence, as opposed to a normally dominant rotational component (Federrath et al. 2008, 2010). Tidal forces can increase the compressive mode too (Renaud et al. 2014). As a result, the probability distribution function for density flattens from an approximately log-normal (Nolan et al. 2015) shape to a broad distribution where a large amount of low-density atomic gas co-exists with a large amount of high-density star-forming gas (Federrath 2013; Federrath \& Klessen 2013; Bournaud et al. 2011b; Renaud et al. 2012). The star formation rate may scale with the mass of the high density gas as usual (e.g., Evans et al. 2014; Clark \& Glover 2014). Physically, this implies a change in the structure of clouds toward more extended HI envelopes around denser $\mathrm{H}_{2}$ and $\mathrm{CO}$ cores.

The molecular envelop traced by $\mathrm{CO}$ in a self-gravitating cloud usually contributes to the gas surface density in the molecular star formation law. Here, the envelopes may be puffed up with high velocity dispersions, giving them low densities and a transparency that keeps them predominantly atomic. Then the envelopes act physically like the CO envelopes of star-forming clouds in non-interacting galaxies, but produce a star formation law with a significant amount of $\mathrm{HI}$ and dark $\mathrm{H}_{2}$ substituted for CO-bright gas.

Another possibility for the HI-rich regions of high SFR is that there is large-scale synchronization of star formation in NGC 2207, starting at perigalacticon $\sim 240$ Myr ago, whereby many of the giant molecular clouds formed in the outer parts have just now dispersed into HI before their OB associations have significantly faded.

We are grateful to Dr. Kartik Sheth for his generous help at all stages of this project. This paper makes use of the following ALMA data: ADS/JAO.ALMA\#2012.1.00357.S. ALMA is a partnership of ESO (representing its member states), NSF (USA) and NINS (Japan), together with NRC (Canada) and NSC and ASIAA (Taiwan) and KASI (Republic of Korea), in cooperation with the Republic of Chile. The Joint ALMA Observatory is operated by ESO, AUI/NRAO and NAOJ. The National Radio Astronomy Observatory is a facility of the National Science Foundation operated under cooperative agreement by Associated Universities, Inc. This research has made use of the NASA/IPAC Extragalactic Database (NED) which is operated by the Jet Propulsion Laboratory, California Institute of Technology, under contract with the National Aeronautics and Space Administration. EB acknowledges support from the UK Science and Technology Facilities Council [grant number ST/M001008/1]. FB acknowledges funding from the EU through grant ERC-StG-257720. 


\section{REFERENCES}

Bigiel, F., Leroy, A., Walter, F., Brinks, E., de Blok, W. J. G., Madore, B., \& Thornley, M. D. 2008, AJ, 136, 2846

Bournaud, F., Chapon, D., Teyssier, R., et al. 2011, ApJ, 730, 4

Bournaud, F., Powell, L. C., Chapon, D., \& Teyssier, R. 2011a, in IAU Symp. 271, Star Formation in Galaxy Mergers: ISM Turbulence, Dense Gas Excess, and Scaling Relations for Disks and Starbursts, ed. N. H. Brummell, A. S. Brun, M. S. Miesch, \& Y. Ponty (Cambridge: Cambridge Univ. Press), 160

Bournaud, F., Daddi, E., Weiss, A., Renaud, F., Mastropietro, C., Teyssier, R. 2015, A\&A, 575, A56

Clark, P.C., \& Glover, S.C.O. 2014, MNRAS, 444, 2396

Dame, T.M., Hartmann, D., \& Thaddeus, P. 2001, ApJ, 655, 863

Elmegreen, B.G., Kaufman, M., \& Thomasson, M. 1993, ApJ, 412, 90

Elmegreen, D.M., Kaufman, M., Brinks, E., Elmegreen, B.G., Sundin, M. 1995, ApJ, 453, 100

Elmegreen, B.G., Sundin, M., Kaufman, M., Brinks, E., Elmegreen, D.M. 1995, ApJ, 453, 139

Elmegreen, B.G., Kaufman, M., Struck, C., et al. 2000, AJ, 120, 630

Elmegreen, D.M., Kaufman, M., Elmegreen, B.G., Brinks, E., Struck, C., Klaric, M., \& Thomasson, M. 2001, AJ, 121, 182

Elmegreen, D.M., Elmegreen, B.G., Kaufman, M., Sheth, K., Struck, C., Thomasson, M., Brinks, E. 2006, ApJ, 642, 158

Evans, N.J., II., Heiderman, A., \& Vutisalchavakul, N. 2014, ApJ, 782, 114

Federrath, C., Klessen, R.S., \& Schmidt, W. 2008, ApJL, 688, L79

Federrath, C., Roman-Duval, J., Klessen, R. S., Schmidt, W., Mac Low, M.-M. 2010, A\&A, 512, A 81

Federrath, C. 2013, MNRAS, 436, 1245

Federrath, C. \& Klessen, R.S. 2013, ApJ, 763, 51 
Kaufman, M., Brinks, E., Elmegreen, B.G., Elmegreen, D.M., Klaric, M., Struck, C., Thomasson, M., Vogel, S. 1999, AJ, 118, 1577

Kaufman, M., Grupe, D., Elmegreen, B. G., Elmegreen, D.M., Struck, C., Brinks, E. 2012, AJ, $144,156 \mathrm{~K}$

Kaufman, M., et al. 2016, in preparation

Kennicutt, R.C., Jr., Calzetti, D., Walter, F., et al. 2007, ApJ, 671, 333

Kennicutt, R.C., Jr., Hao, C.-N., Calzetti, D., Moustakas, J., Dale, D.A., Bendo, G., Engelbracht, C.W., Johnson, B.D., \& Lee, J.C. 2009, ApJ, 703, 1672

Kennicutt, R.C., \& Evans, N.J. 1 2012, ARA\&A, 50, 531

Leroy, A.K., Walter, F., Brinks, E., Bigiel, F., de Blok, W. J. G., Madore, B., \& Thornley, M. D. 2008, AJ, 136, 2782

Leroy, A.K., Bigiel, F., de Blok, W.J.G., et al., 2012, AJ, 144, 3L

Mineo, S., Rappaport, S., Levine, A., Pooley, D., Steinhorn, B., Homan, J. 2014, ApJ, 797, 91

Nolan, C. A., Federrath, C., Sutherland, R. S. 2015, MNRAS, 451, 1380

Powell, L.C., Bournaud, F., Chapon, D., \& Teyssier, R. 2013, MNRAS, 434, 1028

Renaud, F., Kraljic, K., \& Bournaud, F. 2012, ApJL, 760, L16

Renaud, F., Bournaud, F., Kraljic, K., Duc, P.-A. 2014, MNRAS, 442, L33.

Rich, J.A., Kewley, L.J., \& Dopita, M.A. 2015, ApJS, 221, 28

Schlafly \& Finkbeiner 2011, ApJ, 737, 103

Schneider, N., Ossenkopf, V., Csengeri, T., Klessen, R. S., Federrath, C., Tremblin, P., Girichidis, P., Bontemps, S., André, Ph. 2015, A\&A, 575, A79

Smith, P.A., Brand, P.W.J.L., Mountain, C.M., Puxley, P.J., \& Nakai, N. 1991, MNRAS, $252,6 \mathrm{P}$

Struck, C., Kaufman, M., Brinks, E., Thomasson, M., Elmegreen, B.G., \& Elmegreen, D.M. 2005, MNRAS, 364, 69 
Tamburro, D., Rix, H.-W., Leroy, A. K., Mac Low, M.-M., Walter, F., Kennicutt, R. C., Brinks, E., \& de Blok, W. J. G. 2009, AJ, 137, 4424

Ueda, J., Iono, D., Petitpas, G., et al. 2012, ApJ, 745, 65

Velusamy, T., Marsh, K. A., Beichman, C. A., Backus, C. R., \& Thompson, T. J. 2008, AJ, 136, 197

Wetzstein, M., Naab, T., Burkert, A. 2007, MNRAS, 375, 805

Wild, W., Harris, A.J., Eckart, A., et al. 1992, A\&A, 265, 447

This preprint was prepared with the AAS LATEX macros v5.2.

Table 1. Global Properties

\begin{tabular}{lccccc}
\hline \hline Galaxy & $\begin{array}{c}M\left(H_{2}\right) \\
\left(M_{\odot}\right)\end{array}$ & $\begin{array}{c}M(H I)_{\text {total }} \\
\left(M_{\odot}\right)\end{array}$ & $\begin{array}{c}M(H I)_{\text {COfield }} \\
\left(M_{\odot}\right)\end{array}$ & $\begin{array}{c}S_{\nu}(24 \mu m) \\
(\mathrm{mJy})\end{array}$ & $\begin{array}{c}S(H \alpha) \\
\left(\mathrm{erg} \mathrm{cm}^{-2} \mathrm{~s}^{-1}\right)\end{array}$ \\
\hline IC 2163 & $2.1 \times 10^{9}$ & $4.8 \times 10^{9}$ & $2.8 \times 10^{9}$ & $5.9 \times 10^{2}$ & $5.9 \times 10^{-13}$ \\
NGC 2207 & $2.3 \times 10^{9}$ & $2.2 \times 10^{10}$ & $1.3 \times 10^{10}$ & $1.47 \times 10^{3}$ & $2.1 \times 10^{-12}$ \\
\hline
\end{tabular}


Table 2. Locations, Luminosities, and Surface Densities ${ }^{\mathrm{a}}$

\begin{tabular}{|c|c|c|c|c|c|c|c|c|}
\hline ID & $\begin{array}{c}\text { R.A. } \\
(\mathrm{J} 2000) \\
06^{h} 16^{m}\end{array}$ & $\begin{array}{c}\text { Dec. } \\
(\mathrm{J} 2000) \\
-21^{\circ}\end{array}$ & $L(24 \mu \mathrm{m})^{\mathrm{b}}$ & $L(H \alpha)^{\mathrm{c}}$ & $\mathrm{SFR}^{\mathrm{d}}$ & $\Sigma_{\mathrm{SFR}}{ }^{\mathrm{e}}$ & $\Sigma_{\mathrm{H}_{2}}{ }^{\mathrm{e}}$ & $\Sigma_{\mathrm{HI}} \mathrm{e}$ \\
\hline & & IC 2163 & & & & & & \\
\hline $\mathrm{A} 1^{\mathrm{g}, h}$ & 30.612 & 2247.20 & 19.2 & 0.195 & 0.0435 & 0.00748 & 13.6 & 15.6 \\
\hline $\mathrm{A} 2^{\mathrm{h}}$ & 29.789 & 2253.21 & 24.6 & 0.270 & 0.0568 & 0.00977 & 20.6 & 17.2 \\
\hline A3 & 28.858 & 2252.71 & 60.9 & 0.990 & 0.158 & 0.0272 & 35.0 & 9.8 \\
\hline A4 & 27.892 & 2251.21 & 75.9 & 1.16 & 0.193 & 0.0332 & 31.9 & 5.3 \\
\hline A5 & 27.133 & 2243.71 & 93.1 & 2.20 & 0.280 & 0.0482 & 28.1 & 9.5 \\
\hline A6 & 26.345 & 2237.21 & 53.7 & 2.17 & 0.211 & 0.0363 & 7.3 & 10.1 \\
\hline $\mathrm{A} 7^{\mathrm{h}}$ & 30.755 & 2228.20 & 5.7 & 0.101 & 0.0153 & 0.00263 & 2.6 & 15.2 \\
\hline $\mathrm{A} 8$ & 28.930 & 2235.71 & 73.3 & 0.539 & 0.155 & 0.0267 & 19.7 & 7.5 \\
\hline A9 & 28.715 & 2224.21 & 174. & 0.727 & 0.336 & 0.0578 & 36.9 & 11.2 \\
\hline $\mathrm{A} 10^{\mathrm{h}}$ & 28.783 & 2209.21 & 0.15 & 0.086 & 0.0055 & 0.00095 & 0.5 & 12.6 \\
\hline $\mathrm{A} 11^{\mathrm{h}}$ & 28.071 & 2216.71 & 123. & 0.586 & 0.242 & 0.0416 & 52.0 & 13.3 \\
\hline A12 & 27.175 & 2214.71 & 43.3 & 1.11 & 0.135 & 0.0232 & 33.5 & 10.2 \\
\hline A13 & 26.320 & 2212.71 & 52.2 & 1.55 & 0.174 & 0.0299 & 20.7 & 7.1 \\
\hline A14 & 27.072 & 2230.21 & 44.5 & 1.23 & 0.144 & 0.0248 & 15.9 & 7.2 \\
\hline \multirow[t]{2}{*}{ A 15} & 27.999 & 2232.21 & 21.6 & 0.984 & 0.0910 & 0.0157 & 12.6 & 4.2 \\
\hline & & NGC 2207 & & & & & & \\
\hline A16 & 25.135 & 2230.21 & 23.5 & 0.875 & 0.0882 & 0.0162 & 2.0 & 20.9 \\
\hline $\mathrm{A} 17^{\mathrm{h}}$ & 25.386 & 2218.21 & 44.0 & 0.862 & 0.122 & 0.0225 & 14.7 & 27.2 \\
\hline A18 & 24.599 & 2206.71 & 25.3 & 0.576 & 0.0748 & 0.0138 & 14.0 & 17.5 \\
\hline A19 & 24.670 & 2149.21 & 47.3 & 1.13 & 0.143 & 0.0263 & 9.2 & 18.8 \\
\hline $\mathrm{A} 20$ & 23.704 & 2147.71 & 61.0 & 1.02 & 0.160 & 0.0294 & 14.7 & 20.2 \\
\hline A21 & 23.024 & 2141.21 & 92.9 & 1.17 & 0.223 & 0.0410 & 7.6 & 18.9 \\
\hline $\mathrm{A} 22$ & 22.200 & 2139.21 & 47.7 & 0.834 & 0.127 & 0.0234 & 5.5 & 20.9 \\
\hline A23 & 21.091 & 2142.71 & 4.9 & 0.182 & 0.0183 & 0.0034 & 7.4 & 16.4 \\
\hline A24 & 23.381 & 2211.71 & 88.0 & 1.20 & 0.216 & 0.0398 & 12.9 & 12.3 \\
\hline A 25 & 23.167 & 2228.21 & 22.2 & 0.570 & 0.0692 & 0.0127 & 15.9 & 6.2 \\
\hline A26 & 22.450 & 2237.21 & 4.2 & 0.339 & 0.0259 & 0.0048 & 7.5 & 4.5 \\
\hline $\mathrm{A} 27$ & 21.592 & $22 \quad 42.21$ & 15.3 & 0.411 & 0.0487 & 0.0090 & 11.9 & 10.2 \\
\hline A 28 & 20.589 & 2242.21 & 15.2 & 0.513 & 0.0541 & 0.0100 & 12.9 & 15.4 \\
\hline A 29 & 20.519 & $22 \quad 27.71$ & 49.3 & 0.551 & 0.114 & 0.0210 & 9.2 & 8.8 \\
\hline A30 & 19.551 & 2239.71 & 51.5 & 0.736 & 0.128 & 0.0236 & 17.8 & 15.7 \\
\hline A31 & 18.477 & 2233.21 & 24.7 & 0.516 & 0.0705 & 0.0130 & 7.1 & 21.4 \\
\hline A32 & 18.514 & 2154.71 & 96.8 & 1.19 & 0.230 & 0.0423 & 6.3 & 18.2 \\
\hline $\mathrm{A} 33^{\mathrm{h}}$ & 17.225 & 2154.71 & $<0.01$ & 0.090 & 0.0050 & 0.00091 & 0.8 & 27.5 \\
\hline A34 & 17.798 & 2214.21 & 22.9 & 0.670 & 0.0759 & 0.0140 & 2.8 & 19.2 \\
\hline$i^{f}$ & 15.865 & 2202.21 & 866. & 2.39 & 1.606 & 0.296 & 14.9 & 19.9 \\
\hline $\mathrm{A} 36^{\mathrm{h}}$ & 16.580 & $22 \quad 23.21$ & 40.5 & 0.972 & 0.123 & 0.0226 & 4.8 & 28.5 \\
\hline $\mathrm{A} 38^{\mathrm{h}}$ & 17.439 & 2243.71 & 40.7 & 1.07 & 0.128 & 0.0236 & 1.5 & 26.9 \\
\hline A39 & 17.511 & 2255.21 & 17.6 & 0.883 & 0.0786 & 0.0145 & 0.07 & 20.0 \\
\hline A40 & 21.377 & 2310.71 & 44.5 & 0.872 & 0.124 & 0.0228 & 10.3 & 12.0 \\
\hline
\end{tabular}


Table 2-Continued

\begin{tabular}{ccccccccc}
\hline \hline ID & $\begin{array}{c}\text { R.A. } \\
(\mathrm{J} 2000) \\
06^{h} 16^{m}\end{array}$ & $\begin{array}{c}\text { Dec. } \\
(\mathrm{J} 2000) \\
-21^{\circ}\end{array}$ & $L(24 \mu \mathrm{m})^{\mathrm{b}}$ & $L(H \alpha)^{\mathrm{c}}$ & $\mathrm{SFR}^{\mathrm{d}}$ & $\Sigma_{\mathrm{SFR}}{ }^{\mathrm{e}}$ & $\Sigma_{\mathrm{H}_{2}}{ }^{\mathrm{e}}$ & $\Sigma_{\mathrm{HI}}{ }^{\mathrm{e}}$ \\
\hline $\mathrm{A} 41$ & 23.740 & 2259.21 & 8.7 & 0.520 & 0.0433 & 0.0080 & 5.7 & 13.5 \\
$\mathrm{~A} 37$ & 23.811 & 2201.21 & 20.5 & 0.774 & 0.0776 & 0.0143 & 8.7 & 14.8 \\
$\mathrm{~A} 42$ & 20.589 & 2213.71 & 2.2 & 0.136 & 0.0112 & 0.0022 & 5.3 & 7.4 \\
$\mathrm{~A} 43$ & 19.122 & 2256.71 & 4.8 & 0.305 & 0.0249 & 0.0046 & 3.2 & 12.8 \\
$\mathrm{~A} 35$ & 19.301 & 2147.21 & 16.1 & 0.375 & 0.0481 & 0.0089 & 5.2 & 14.0 \\
\hline
\end{tabular}

a The units are as follows: R.A. in seconds, Dec. in arcminutes and arcseconds; $L(24 \mu \mathrm{m})$ and $L(\mathrm{H} \alpha)$ in $10^{40} \mathrm{erg} \mathrm{s}^{-1}$; $\mathrm{SFR}$ in $\mathrm{M}_{\odot} \mathrm{yr}^{-1} ; \Sigma_{\mathrm{SFR}}$ in $\mathrm{M}_{\odot} \mathrm{pc}^{-2} \mathrm{Myr}^{-1} ; \Sigma_{\mathrm{H}_{2}}$ and $\Sigma_{\mathrm{HI}}$ in $\mathrm{M}_{\odot} \mathrm{pc}^{-2}$.

${ }^{\mathrm{b}} L(24 \mu \mathrm{m})$ is $\nu L_{\nu}$ at $24 \mu \mathrm{m}$.

${ }^{\mathrm{c}} L(\mathrm{H} \alpha)$ is corrected for Galactic foreground extinction using $A_{\mathrm{V}} / 1.28=0.186$ mag. $L(H \alpha)$ for apertures A3, A4, A5, A6, A12, A13, A14, and A15 have also been corrected for foreground extinction by the outer arm of NGC 2207 in front of IC 2163. The latter correction (see text) amounts to an average of $0.85 \pm 0.43$ mag at $H \alpha$.

${ }^{\mathrm{d}} \mathrm{SFR}\left(\mathrm{M}_{\odot} \mathrm{yr}^{-1}\right)=5.5 \times 10^{-42}[L(H \alpha)+0.031 L(24 \mu \mathrm{m})]\left(\mathrm{erg} \mathrm{s}^{-1}\right)$.

${ }^{\text {e }}$ Surface densities have been corrected to face-on using $i=40^{\circ}$ for IC 2163 and $i=35^{\circ}$ for NGC 2207.

${ }^{\mathrm{f}}$ Feature $i$

gApertures are $14^{\prime \prime}$ in diameter.

${ }^{\mathrm{h}}$ In IC 2163, apertures A1 and A2 are on parts of the massive HI cloud I3, A7 is on I2, and A10 and A11 are on parts of I5. In NGC 2207, apertures A17, A33, A36, and A38 are on massive HI clouds N6, N2, N3, and N4, respectively. 

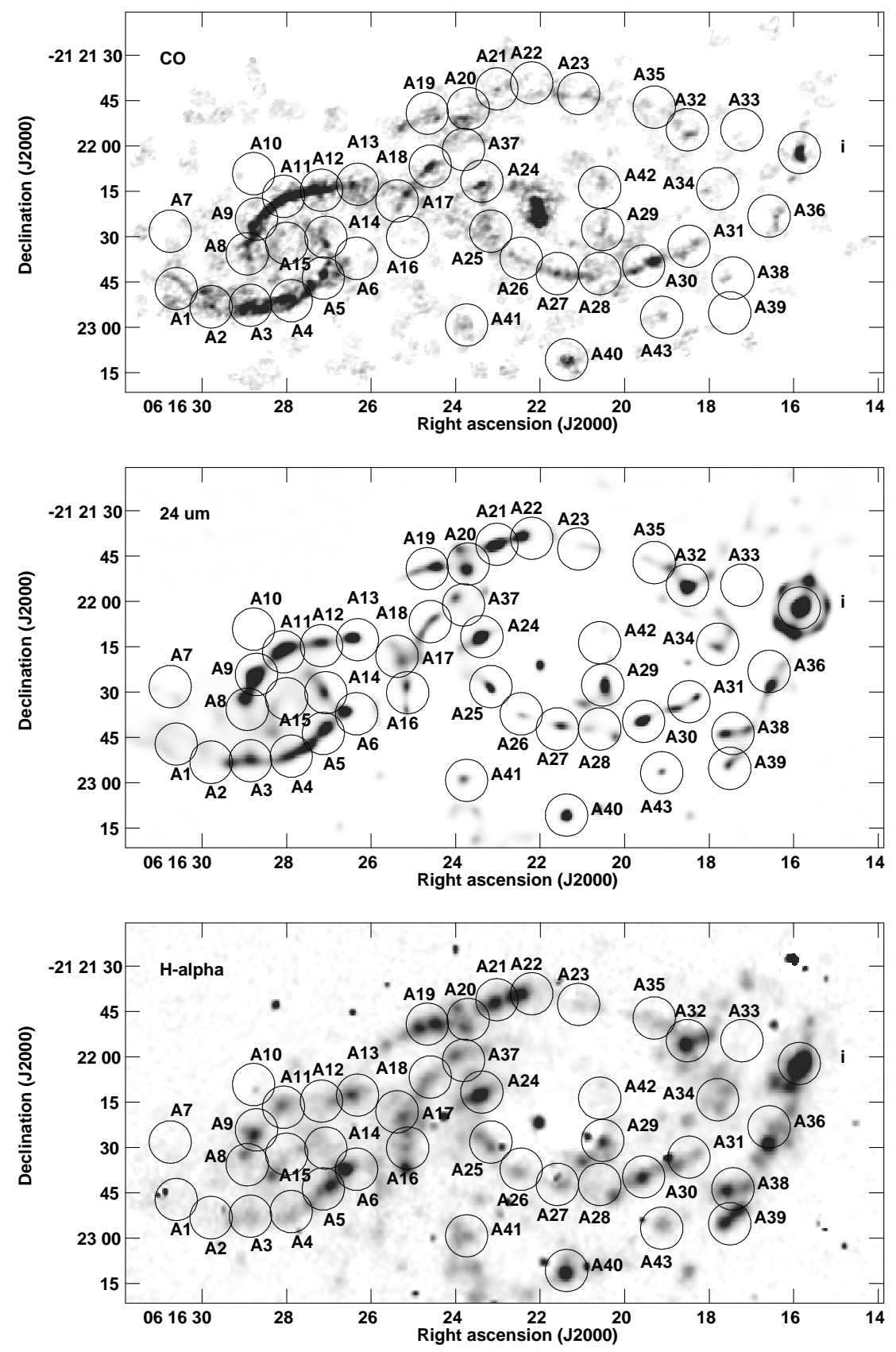

Fig. 1.- (top) Integrated CO intensity (middle) HiRes $24 \mu \mathrm{m}$ flux density, and (bottom) $\mathrm{H} \alpha$ (uncorrected for extinction) with regions of star formation considered here. The aperture size is $14^{\prime \prime}=2.4 \mathrm{kpc}$ in diameter. 

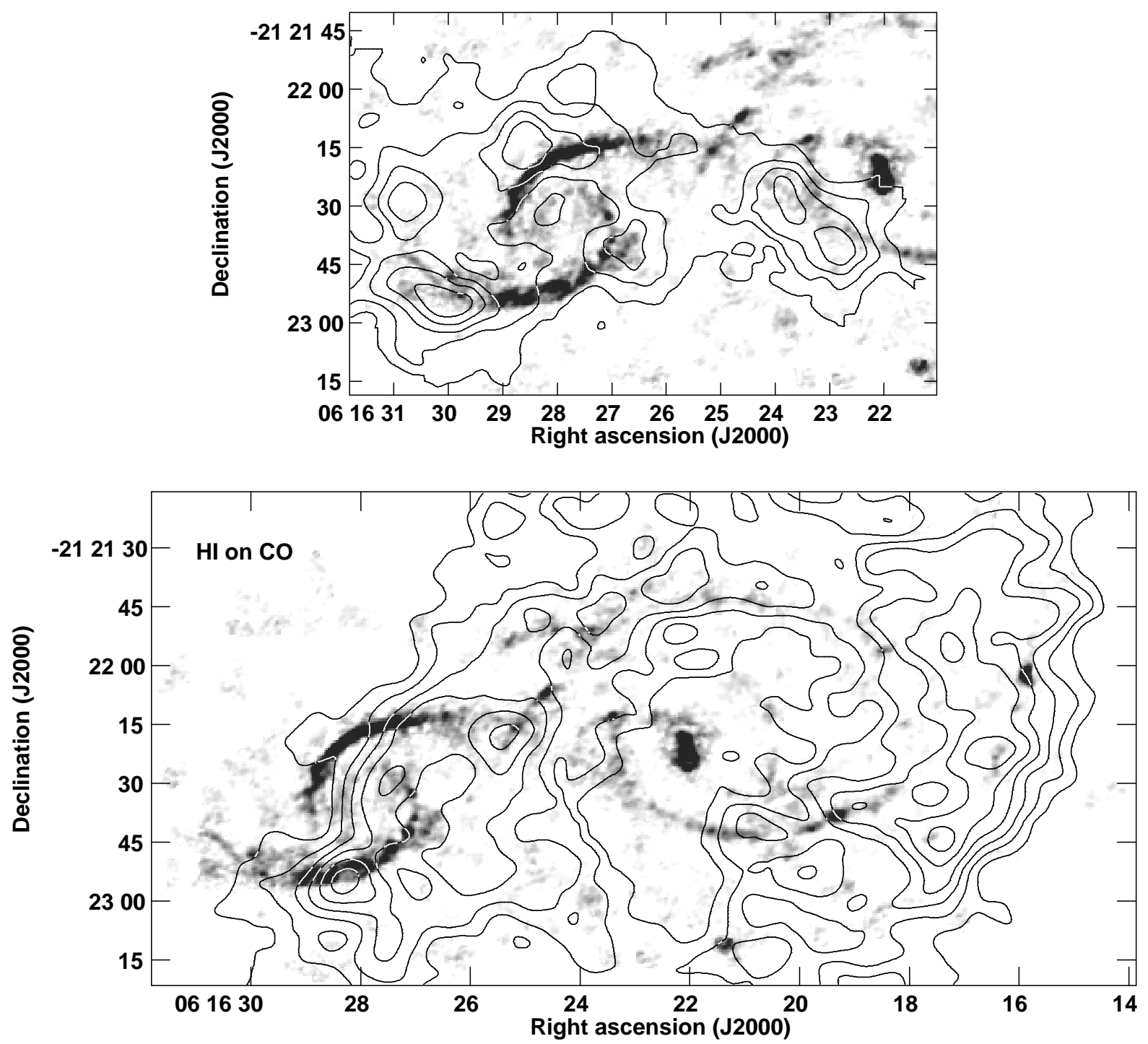

Fig. 2.- (top) HI column density contours superposed on CO for IC 2163. The contours are at line-of-sight $N(H I)=5,10,15,20$, and $25 M_{\odot} \mathrm{pc}^{-2}$. (bottom) HI on NGC 2207. The contours are at 10, 15, 20, 25, 30, $35 M_{\odot} \mathrm{pc}^{-2}$, with $5 M_{\odot} \mathrm{pc}^{-2}$ omitted for clarity. The HI contributions to each galaxy were determined on the basis of their kinematics. 

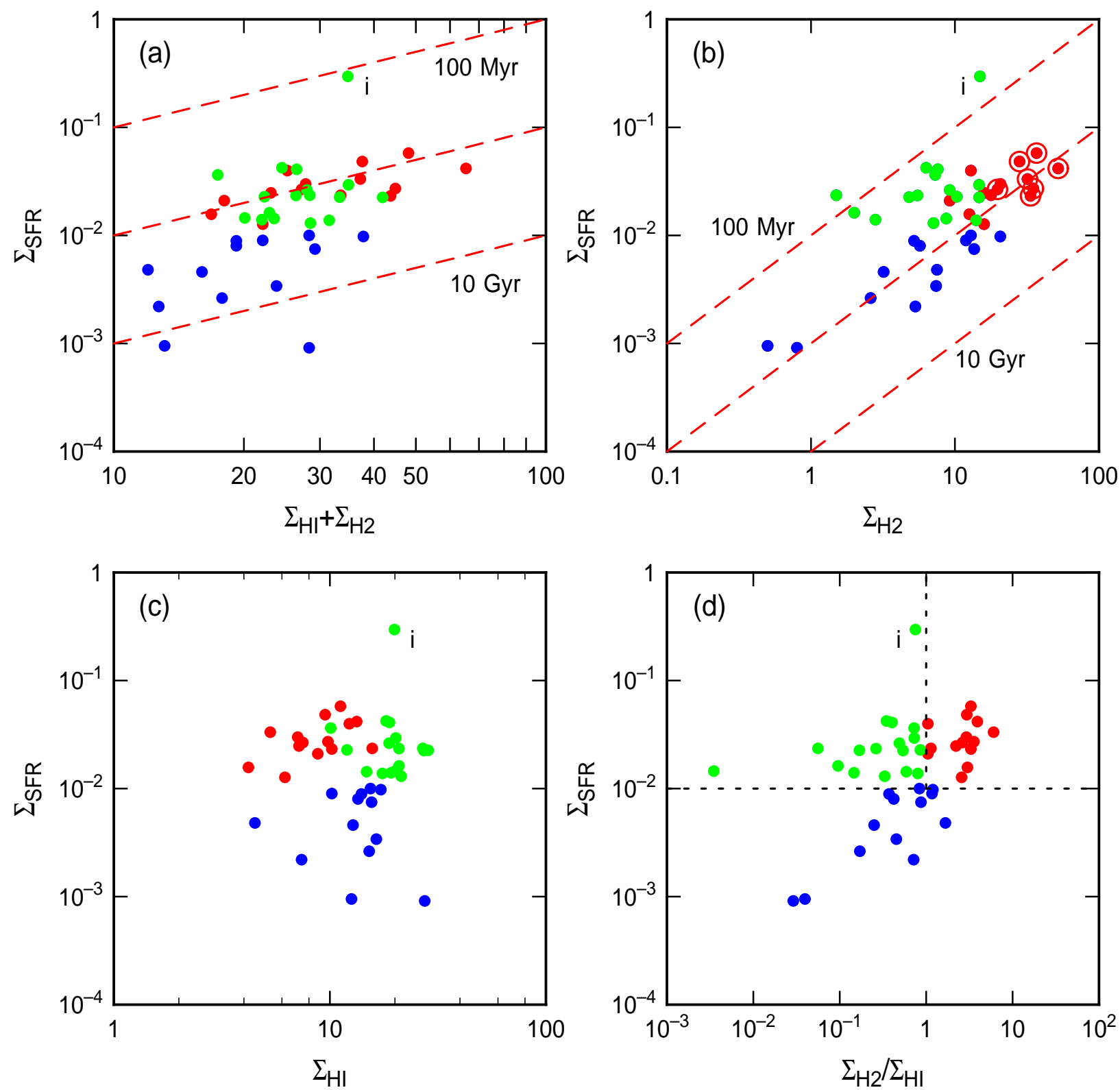

Fig. 3.- The SFR surface density is plotted versus the gas surface density in various forms. Color indicates regions with high SFR (green for low molecular fraction and red for high molecular fraction) and low SFR (blue), as indicated in panel (d). Feature $i$ is labeled. The red circles in (b) are for locations in the ocular rim of IC 2163. The red dashed lines in (a) and (b) indicate gas consumption times. The dashed lines in (d) separate regions of high and low SFR and high and low $\Sigma_{\mathrm{H} 2} / \Sigma_{\mathrm{HI}}$ ratio. 


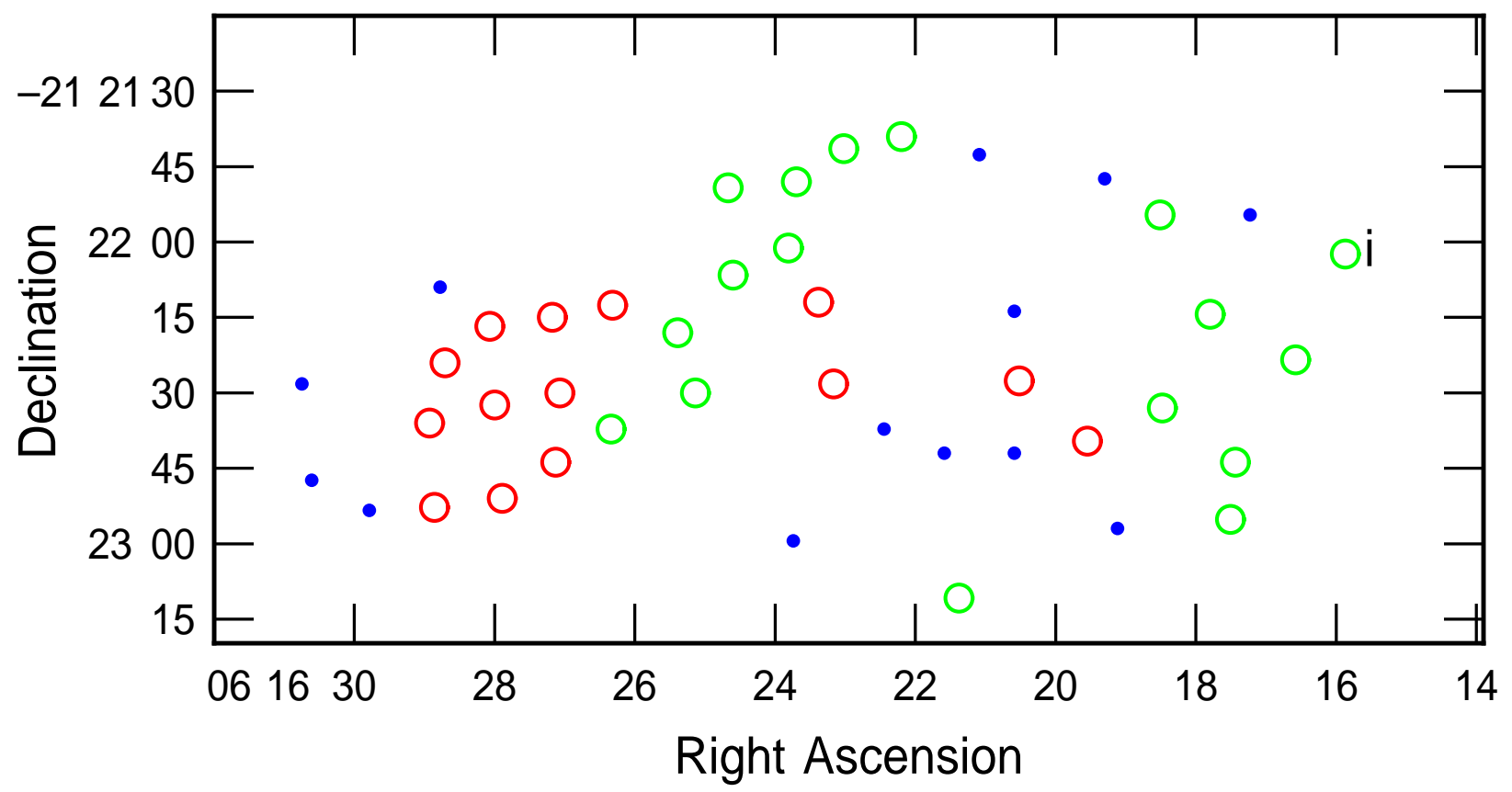

Fig. 4.- Positions of the selected regions with color coding as in Figure 2. The green regions have high SFRs yet are dominated by atomic gas. Feature $i$ is on the right. 